\title{
Creating a pandemic of prediabetes: the proposed new diagnostic criteria for impaired fasting glycaemia
}

\author{
K. Borch-Johnsen ${ }^{1}$ S. Colagiuri ${ }^{2} \cdot$ B. Balkau ${ }^{3}$ C. Glümer ${ }^{1,4} \cdot$ B. Carstensen ${ }^{1} \cdot$ A. Ramachandran ${ }^{5}$ Y. Dong ${ }^{6}$. \\ W. Gao ${ }^{6}$ \\ 1 Steno Diabetes Center, Gentofte, Denmark \\ 2 Department of Endocrinology and Diabetes, Prince of Wales Hospital, Sydney, Australia \\ 3 INSERM Unit 258-IFR69, Cardiovascular and Metabolic Epidemiology, Villejuif, France \\ ${ }^{4}$ Research Centre for Prevention and Health, Glostrup, Denmark \\ ${ }^{5}$ Diabetes Research Centre, M.V. Hospital for Diabetes, Chennai, India \\ ${ }^{6}$ Qingdao Epidemiology Study Group, Qingdao Endocrinology Diabetes Institute \& Hospital, Qingdao, China
}

\section{Abstract}

Aims/hypothesis. In November 2003 the American Diabetes Association expert committee on the diagnosis and classification of diabetes mellitus suggested a revision of the diagnostic criteria for IFG, lowering the diagnostic threshold from 6.1 to $5.6 \mathrm{mmol} / \mathrm{l}$. The aim of the present study was to evaluate the consequences of this change with respect to: (i) the prevalence of IFG in five different countries; (ii) the concordance between IFG and IGT (classification of individuals); and (iii) the cardiovascular risk profile of these groups. Finally we discuss the likelihood that intervention for cardiovascular risk and prevention strategies developed for individuals with IGT are applicable to subjects with IFG.

Methods. The first part of the study is based on the population-based Danish Inter99 study, where 6265 individuals, aged 30 to 60 years and without previously diagnosed diabetes, underwent an oral glucose tolerance test. The second part is based on the DETECT-2 project, in which studies from China, India, France and USA were used to analyse the impact of the proposed revision of the diagnostic criteria in different ethnic groups.

Results. The proposed change in diagnostic criteria would increase the prevalence of IFG in Denmark from
11.8 to $37.6 \%$. The proposed IFG category would identify $60.0 \%$ of all subjects with IGT compared to $29.2 \%$ with the old criteria, but among individuals with the new IFG category only $18.5 \%$ would also have IGT. Individuals with isolated IFG had lower insulin levels and a lower cardiovascular risk profile with the proposed criteria compared with the current WHO criteria. Data from the DETECT-2 study confirmed the marked increase in the prevalence of IFG, and the estimated number of individuals in the age range 40 to 64 years with IFG in urban India, urban China and the USA would increase by $78 \%, 135 \%$ and $193 \%$ respectively.

Conclusions/interpretation. The proposed revised diagnostic criteria will lead to a dramatic increase in the prevalence of IFG, but the concordance rate between IFG and IGT remains low. This new IFG group will have a more favourable cardiovascular risk profile than the current IFG group as defined by the WHO. This seriously questions whether the existing intervention strategies are applicable to the new category of individuals with IFG.

Keywords Cardiovascular risk profile - Diagnosis · Fasting glucose $\cdot$ Impaired fasting glucose $\cdot$ Impaired fasting glycaemia $\cdot$ Impaired glucose tolerance
Received: 6 February 2004 / Accepted: 14 May 2004

Published online: 24 July 2004

(C) Springer-Verlag 2004

K. Borch-Johnsen (

Steno Diabetes Center, Niels Steensensvej 2,

2820 Gentofte, Denmark

E-mail: kbjo@steno.dk

Tel.: +45-4443-9415, Fax: +45-4443-8232

Abbreviations: ADA, American Diabetes Association · FPG, fasting plasma glucose $\cdot$ NHANES III, Third National Health and Nutrition Survey · WHO, World Health Organization

\section{Introduction}

The American Diabetes Association (ADA) expert committee on the diagnosis and classification of diabetes mellitus recently published a follow-up report [1]. This report follows only 6 years after the last expert committee report [2] and only 4 years after the recommendations from the World Health Organization (WHO) [3]. The principle changes in the new ADA recommendations are a lowering of the threshold for 
normal fasting plasma glucose from 6.1 to $5.6 \mathrm{mmol} / \mathrm{l}$ and redefinition of IFG as fasting plasma glucose from 5.6 to $6.9 \mathrm{mmol} / \mathrm{l}$. The rationale for lowering the diagnostic threshold has been questioned on the basis of data from NHANES III $[4,5]$.

The major reason for redefining IFG was an attempt to improve the alignment of IFG and IGT, the corresponding intermediate category based on the oral glucose tolerance test, and through this improve predictions with regard to future development of Type 2 diabetes. The proposed new diagnostic threshold is derived from receiver operator characteristic curves of the different levels of fasting plasma glucose which predict the development of diabetes. The optimal cutpoint (optimising the sum of sensitivity and specificity) was between 5.2 and $5.7 \mathrm{mmol} / \mathrm{l}$ [1]. A secondary, but equally important consideration was to increase the proportion of individuals with IGT who would also be identified as having IFG. With the current definition [2], only $29 \%$ of individuals with IGT also have IFG [6]. The identification of patients with IGT is important from a preventive medicine perspective, as this is the group where intervention studies have proven effective in preventing or delaying progression to diabetes $[7,8,9,10]$.

The 1997 ADA expert committee report was followed by a thorough analysis of existing epidemiological data analysing the consequences of changing the diagnostic criteria. The DECODE study [6], under the auspices of the European Diabetes Epidemiology Group, was established with the sole aim of evaluating the consequences of the revised diagnostic criteria, and together with many other studies, concluded that IFG and IGT differed considerably with respect to prognosis (all-cause mortality, cardiovascular disease morbidity and mortality) $[11,12]$ and the distribution of cardiovascular risk factors [6]. Based on this experience, it would seem prudent to use the extensive existing data to assess the consequences of changing diagnostic criteria before new criteria are actually proposed, thus providing evidence-based rather than opinion-based recommendations. The 2003 follow-up report [1] has done this to a larger extent than the previous one [2], but still leaves several important questions unaddressed:

1. What is the concordance between IFG and IGT with respect to classification of individuals?

2. What is the cardiovascular risk profile of these groups?

3. What impact will the lowering of the diagnostic threshold for IFG have on the prevalence of IFG worldwide?

4. What are the implications for the implementation of evidence-based preventative strategies $[7,8,9,10]$ ?

The aim of the present study was to answer the first three questions by drawing on five large population- based or otherwise representative epidemiological studies from China, Denmark, France, India and USA, all of which form part of the DETECT-2 dataset [13]. Our study also discusses the last question with reference to the results of previously conducted prevention trials $[7,8,9,10]$. As the other studies did not have one or more of the parameters included in the analysis, it was only possible to study the impact of the change in diagnostic criteria on the cardiovascular risk profile in the Danish Inter99 study [14].

\section{Subjects and methods}

The Inter99 study. The Inter99 study is a population-based primary prevention study on cardiovascular disease and Type 2 diabetes mellitus. The study population was all 61,301 individuals born in the years 1939 to 1940,1944 to 1945,1949 to 1950,1954 to 1955,1959 to 1960,1964 to 1965 , or 1969 to 1970 , and living in eleven municipalities in the south-western part of Copenhagen County. These people were identified from the Civil Registration System. An age- and sex-stratified random sample of 13,016 individuals was drawn, of whom 6784 participated. Baseline data were collected from March 1999 until January 2001. The recruitment and baseline characteristics have been described elsewhere [14]. All individuals participating in the survey completed a questionnaire. After an overnight fast, participants underwent various procedures including anthropometric measurements, blood sampling, and a standard 75-g standard OGTT. Participants with previously diagnosed diabetes had fasting plasma glucose measured, but did not have an OGTT. Plasma glucose was sampled in a heparin-NaF tube. The samples were put on ice immediately and centrifuged within 30 minutes. The glucose was analysed using the hexokinase/G6P-DH (Boehringer Mannheim, Germany). All participants gave written consent and the protocol was in accordance with the Helsinki declaration and approved by the local ethical committee.

The DETECT-2 project. The DETECT-2 project is an international data pooling collaboration specifically designed to address a number of issues relating to screening and early detection of Type 2 diabetes and IGT, with a special emphasis on the impact of ethnicity and population differences on screening protocols. DETECT-2 is overseen by an international steering committee with the data pooling and analysis being performed at the Steno Diabetes Center, Gentofte, Denmark. The broad questions which DETECT-2 is investigating include: (i) comparison and evaluation of selected strategies for screening and early detection of undiagnosed Type 2 diabetes and IGT across a range of populations from diverse ethnic backgrounds; (ii) the development of a simple screening strategy for Type 2 diabetes and IGT that would be applicable to different populations throughout the world; and (iii) an assessment of the implications with regard to morbidity and mortality for individuals categorised on the basis of a screening program for diabetes. Centres from around the world have supplied datasets. The minimum requirements for participation in DETECT-2 were population-based surveys that included information on at least 500 people or surveys of large cohorts of employees, in both of which all participants without previously known diabetes had a 75-g OGTT.

Individual data were sent to the Steno Diabetes Center, Copenhagen, Denmark. For each subject studied the following data were used in this analysis: sex, age, height, weight, status 
Table 1. Characteristics of the study populations in each of the five studies included

\begin{tabular}{llllll}
\hline & Inter99 [14] & Paris Prospective [15] & Qingdao [16] & NUDS [17] & NHANES [18] \\
\hline Country & Denmark, & France, & Urban China, & Urban India, & USA, \\
& $n=6265$ & $n=7034$ & $n=1808$ & 039 & $4=3517$ \\
Percentage males & 48.6 & 100 & 37.2 & 47.0 & 48.8 \\
Age range (years) & $30-61$ & $44-55$ & $30-74$ & $40-99$ & $40.4(14.2)$ \\
Mean age in years & $46.0(7.9)$ & $49.0(2.0)$ & $53.7(10.7)$ & $23.1(7.9)$ & $27.8(5.5)$ \\
Mean BMI in kg/m & $26.3(4.6)$ & $26.0(3.3)$ & $26.4(3.6)$ & 15.0 & 11.2 \\
\% Diabetes & 4.1 & 4.4 & 10.3 & 10.6 & 20.3 \\
\% IGT & 12.0 & 8.2 & 11.2 & 37.6 & 9.5 \\
\% IFG (1999 WHO)* & 11.8 & 15.9 & 26.7 & 28.5 \\
\% IFG (2003 ADA)\# & 37.6 & 45.2 & & \\
\hline
\end{tabular}

Values are means (SD), except where percentages or ranges are given. * Fasting plasma glucose 6.1-6.9 mmol/1; \# fasting plasma glucose 5.6-6.9 mmol/l. NHANES, National Health and Nutrition Survey; NUDS, National Urban Diabetes Survey

Table 2. The distribution of subjects by fasting and 2-h plasma glucose in the Inter99 study

2-hour plasma glucose

$<7.8 \mathrm{mmol} / \mathrm{l}$

3348

1387

520

26

5281
7.8-11.0 mmol/1

$\geq 11.1 \mathrm{mmol} / 1$

FPG

$<5.6 \mathrm{mmol} / 1$
$5.6-6.0 \mathrm{mmol} / 1$

$6.1-6.9 \mathrm{~mm}$
$\geq 7.0 \mathrm{mmol} / \mathrm{l}$

Total

302
230
219
43
794

27
28
49
86
190

3677

1645

788

155

6265

FPG, fasting plasma glucose

of known diabetes and fasting and 2-h blood glucose. Details were also provided on the blood specimen used for the glucose assay (venous whole blood, venous plasma, capillary whole blood) and the method of glucose assay. To allow for the different methods of blood glucose measurement, the WHO equivalence table was used to convert all results into plasma glucose equivalents [3].

For the present analysis the following five studies were included; the INTER99 study [14], the Paris Prospective Study [15], the Qingdao study from China [16], the National Urban Diabetes Study (NUDS) from India [17] and the Third National Health and Nutrition Survey (NHANES III) from the US [18]. Basic characteristics of each of the five study populations are given in Table 1.

To estimate the impact of the proposed change in diagnostic criteria on the total number of individuals with IFG in the three largest countries (India, China and USA), the age-specific prevalence of IFG for the age intervals 40 to 54 and 55 to 64 years was calculated on the basis of the above studies [16, 17, 18]. Population data on the number of individuals in each of these age categories were taken from the website of the United Nations Statistical Office (http://www.un.org/esa/population/ unpop.htm) [19]. As the prevalence of diabetes, IGT and IFG is higher in urbanised areas than in rural areas and the surveys in India and China covered urbanised areas only, the analysis for these two countries was restricted to the proportion of the population living in urbanised areas $38.6 \%$ in China and $28.3 \%$ in India [19]). For USA the entire population was used, as NHANES III is a representative population-based sample.

All individuals without previously diagnosed diabetes were classified according to the fasting and 2-hour glucose value during an OGTT using both the current WHO criteria for diabetes, IGT and IFG [3] and the new proposed revised ADA criteria [1]. The WHO criteria based on fasting plasma glucose (FPG) are: normal: $\leq 6.0 \mathrm{mmol} / \mathrm{l}$; IFG: $6.1-6.9 \mathrm{mmol} / \mathrm{l}$; diabetes: $\geq 7.0 \mathrm{mmol} / \mathrm{l}$. The corresponding, proposed revised ADA criteria are: normal: $\leq 5.5 \mathrm{mmol} / \mathrm{l}$; IFG: 5.6-6.9 mmol/l; diabetes: $\geq 7.0 \mathrm{mmol} / \mathrm{l}$. The WHO criteria and revised ADA criteria based on 2-hour plasma glucose are identical, namely: normal: $<7.8 \mathrm{mmol} / \mathrm{l}$; IGT: $7.8-11.0 \mathrm{mmol} / \mathrm{l}$; diabetes $\geq 11.1 \mathrm{mmol} / \mathrm{l}$.

All analyses were performed at the Steno Diabetes Center using SAS 8-2 (SAS Institute, Cary, N.C., USA) and R 1.8 (R development Core Team, Vienna, Austria). In each centre, data collection was performed according to local ethical rules and according to the Helsinki Declaration.

\section{Results}

Concordance between IFG and IGT and cardiovascular risk profile of persons in these groups (Inter99). Table 2 shows the distribution of the 6265 individuals without previously diagnosed diabetes and with valid data on fasting and 2-hour OGTT plasma glucose. Of the participants, $12.0 \%$ had a 2 -hour glucose value in the IGT range. The prevalence of IFG increased from $11.8 \%$ (WHO criteria) to $37.6 \%$ using the proposed revised ADA criteria.

The combined prevalence of IFG and/or IGT was $20.3 \%(1271 / 6265)$ according to the WHO criteria, 
Table 3. The cardiovascular risk profile according to the diagnostic criteria in the Inter99

\begin{tabular}{|c|c|c|c|}
\hline & \multicolumn{2}{|l|}{ IFG } & \multirow[t]{2}{*}{$p$ value } \\
\hline & 1999 WHO criteria a & $\begin{array}{l}\text { Additional subjects diagnosed } \\
\text { by } 2003 \text { ADA criteria }{ }^{b}\end{array}$ & \\
\hline Number & $n=788$ & $n=1645$ & \\
\hline$\%$ Women & 29.6 & 38.4 & $<0.0001$ \\
\hline Age in years & $49.4(6.8)$ & $47.4(7.4)$ & $<0.0001$ \\
\hline Total cholesterol (mmol/l) & $5.9(1.2)$ & $5.7(1.1)$ & $<0.0001$ \\
\hline HDL cholesterol (mmol/l) & $1.3(0.4)$ & $1.4(0.4)$ & 0.002 \\
\hline Triglyceride $^{\mathrm{c}}(\mathrm{mmol} / \mathrm{l})$ & $1.5(0.6)$ & $1.2(0.5)$ & $<0.0001$ \\
\hline Fasting insulin ${ }^{\mathrm{c}}(\mathrm{pmol} / \mathrm{l})$ & $47.0(0.6)$ & $37.8(0.5)$ & $<0.0001$ \\
\hline 2 -h insulinc $(\mathrm{pmol} / \mathrm{l})$ & $211.2(0.9)$ & $160.1(0.9)$ & $<0.0001$ \\
\hline BMI $\left(\mathrm{kg} / \mathrm{m}^{2}\right)$ & $28.4(4.8)$ & $27.0(4.4)$ & $<0.0001$ \\
\hline
\end{tabular}

Values are means (SD), except where percentages are given. a $6.1-6.9 \mathrm{mmol} / \mathrm{l} ;{ }^{\mathrm{b}}$ 5.6-6.0 mmol/1; ${ }^{\mathrm{c}}$ Values are geometric means and coefficient of variation. DBP, diastolic blood pressure; SBP, systolic blood pressure

Table 4. The prevalence of IGT, IFG, combined IFG and IGT and prevalence of IGT among IFG individuals according to the two sets of criteria ${ }^{\mathrm{a}}$

\begin{tabular}{|c|c|c|c|c|c|c|c|c|}
\hline \multirow[t]{2}{*}{ Country } & \multirow[t]{2}{*}{$\begin{array}{l}\text { Total } \\
\text { number }\end{array}$} & \multirow{2}{*}{$\begin{array}{l}\text { Prevalence of IGT } \\
\mathrm{WHO}+\mathrm{ADA}(\%)\end{array}$} & \multicolumn{2}{|c|}{ Prevalence of IFG } & \multicolumn{2}{|c|}{$\begin{array}{l}\text { Prevalence, combined } \\
\text { IFG and IGT }\end{array}$} & \multicolumn{2}{|c|}{$\begin{array}{l}\text { Proportion of IFG } \\
\text { individuals who have IGT }\end{array}$} \\
\hline & & & WHO (\%) & $\operatorname{ADA}(\%)$ & WHO (\%) & $\operatorname{ADA}(\%)$ & WHO (\%) & $\operatorname{ADA}(\%)$ \\
\hline Denmark & 6265 & 12.0 & 12.6 & 38.8 & 3.5 & 7.2 & 27.8 & 18.5 \\
\hline France & 7034 & 8.2 & 16.3 & 45.7 & 3.5 & 6.1 & 21.6 & 13.4 \\
\hline India & 10039 & 11.2 & 11.0 & 38.6 & 3.0 & 7.2 & 26.7 & 18.8 \\
\hline
\end{tabular}

a existing 1999 WHO criteria for IFG and IGT and the proposed new 2003 ADA criteria

and among these, 219 (17.2\%) had both IGT and IFG. For the proposed revised ADA criteria the prevalence of IFG and/or IGT was 42.4\% (2658/6265), and among these, 449 (16.9\%) had both IGT and IFG. Using the WHO criteria for IFG, this category would identify $29.2 \%(219 / 751)$ of individuals with IGT, while the proposed revised ADA criteria would identify $56.5 \%$ (449/751). However, among individuals with IFG by the WHO criteria $27.8 \%(219 / 788)$ also had IGT, while $6.2 \%$ (49/788) had previously undiagnosed diabetes. Using the proposed new ADA criteria for IFG the corresponding figures would be $18.5 \%$ (449/2433) for persons who also had IGT and 3.1\% (77/2433) for those with previously undiagnosed diabetes. Although fasting plasma glucose as well as 2-hour glucose increased with age and BMI, neither of these factors modified the association between FPG and 2-hour plasma glucose.

The effect of lowering the diagnostic threshold for IFG on the cardiovascular risk profile was analysed comparing the cardiovascular risk profile in individu- als with IFG according to the current WHO definition with that of those additional cases of IFG identified by lowering the diagnostic threshold using the new proposed ADA definition. Individuals with previously diagnosed diabetes were excluded from the analysis, but otherwise the analysis was performed without considering the 2-hour plasma glucose result. As shown in Table 3, the additional individuals identified with IFG by the proposed new ADA criteria had a more favourable cardiovascular risk profile with significantly lower mean age, blood pressure, total cholesterol, triglycerides, fasting insulin, 2-hour insulin levels, as well as BMI and waist circumference (all $p<0.001$ ), and significantly higher HDL cholesterol $(p<0.002)$.

Effect of lower diagnostic threshold for IFG on the prevalence of IFG (DETECT-2). The results from all five studies from the DETECT-2 project are summarised in Table 4. In all studies the proposed new ADA criteria increase the prevalence of IFG two- to four-fold. The percentage of individuals with com- 
Table 5. The prevalence and estimated number of individuals with IFG according to the two sets of criteria ${ }^{\mathrm{a}}$ in the 40 to 64 age group in urban India, urban China and the USA ${ }^{b}$

\begin{tabular}{|c|c|c|c|c|c|c|c|}
\hline & \multicolumn{3}{|l|}{ 40-54 years } & \multicolumn{3}{|l|}{ 55-64 years } & \multirow{2}{*}{$\begin{array}{l}\text { 40-64 years } \\
\text { Number with } \\
\text { IFG (million) }\end{array}$} \\
\hline & $\begin{array}{l}\text { Population } \\
\text { (million) }\end{array}$ & $\begin{array}{l}\text { Prevalence } \\
\text { of IFG }(\%)\end{array}$ & $\begin{array}{l}\text { Number with } \\
\text { IFG (million) }\end{array}$ & $\begin{array}{l}\text { Population } \\
\text { (million) }\end{array}$ & $\begin{array}{l}\text { Prevalence } \\
\text { of IFG (\%) }\end{array}$ & $\begin{array}{l}\text { Number with } \\
\text { IFG (million) }\end{array}$ & \\
\hline India & 41.1 & & & 16.7 & & & \\
\hline 1998 WHO & & 29.6 & 12.2 & & 29.0 & 4.8 & 17.0 \\
\hline Proposed 2003 ADA & & 52.7 & 21.7 & & 50.9 & 8.5 & 30.2 \\
\hline China & 90.2 & & & 34.3 & & & \\
\hline 1998 WHO & & 10.2 & 9.2 & & 16.9 & 5.8 & 15.0 \\
\hline Proposed 2003 ADA & & 26.4 & 23.8 & & 33.5 & 11.5 & 35.3 \\
\hline USA & 61.3 & & & 24.6 & & & \\
\hline 1998 WHO & & 8.6 & 5.3 & & 12.9 & 3.2 & 8.5 \\
\hline Proposed 2003 ADA & & 26.0 & 16.0 & & 36.2 & 8.9 & 24.9 \\
\hline
\end{tabular}

a Existing 1999 WHO criteria for IFG and IGT and the proposed new 2003 ADA criteria; ${ }^{\text {b }}$ determined using population-based surveys from India [17], China [16] and the USA [18]

bined IFG and IGT increases by 74 to $140 \%$ but the percentage of people with IFG who also have IGT decreases by 17 to $38 \%$.

Age-specific prevalence rates were used to estimate the regional impact of the proposed revised ADA criteria in India, China and the USA. For China and India only the populations living in urbanised areas were included. As shown in Table 5, changing the diagnostic criteria for IFG would increase the total number of individuals with IFG in the 40- to 64-year age range from 17.0 to 30.2 million in urban India $(77 \%$ increase), from 15.0 to 35.3 million in urban China (135\% increase) and from 8.4 to 24.9 million in USA (196\% increase).

\section{Discussion}

The introduction of IFG as a new category of glucose intolerance was part of the 1997 recommendations of the ADA expert committee on the diagnosis and classification of diabetes mellitus [2]. The aim was to define glucose intolerance in a way that made it identifiable on the basis of fasting plasma glucose, thereby creating a category that is analogous with IGT, as defined on the basis of 2-h glucose following an OGTT. It became clear that these two categories did not identify the same individuals [11] and they did not have the same impact on morbidity, mortality and cardiovascular risk profile $[6,12]$. However, both IFG and IGT do predict subsequent development of diabetes [20, 21], confirming their role as pre-diabetic states. In the present study we have reviewed the impact of the ADA's proposed change in diagnostic criteria for IFG with respect to the prevalence of IFG, the concordance between IFG and IGT, the cardiovascular risk profiles and the likely impact on prevention strategies.

Using the current WHO criteria, IFG has generally been reported to have a lower prevalence than IGT
[21]. In the Inter99 study we found that the prevalence of IFG and IGT in the age-group 30 to 60 years was almost identical, between 12 and $13 \%$. Lowering the diagnostic threshold to $5.6 \mathrm{mmol} / \mathrm{l}$ increased the prevalence of IFG to $38.8 \%$ or more than 3 times higher than the prevalence of IGT. With the revised criteria more than one out of three persons would be labelled "prediabetic", and only 59\% of the population in the 30 to 60 age-range would have normal glucose tolerance based on fasting plasma glucose.

For the studies included in the DETECT-2 project a similar pattern was found. Using the current WHO criteria, the prevalence of IFG was higher than IGT in a French male population, equal to IGT prevalence in China and India, and lower than IGT in the USA. A lowering of the diagnostic threshold to $5.6 \mathrm{mmol} / \mathrm{l}$ would increase the prevalence of IFG to between $28.7 \%$ (China) and $45.7 \%$ (France).

The 1997 definition of IFG and IGT showed a rather poor concordance rate with only 20 to $30 \%$ of all individuals with IGT being identified by the IFG category [22]. In the INTER99 study $29 \%$ of all IGT cases were included in the IFG category. This observation was one of the key arguments used by the 2003 ADA expert committee to justify the proposed lowering of the diagnostic criteria for IFG. With the proposed new, lower IFG threshold, $60 \%$ of all individuals with IGT are identified, and thus the lowering doubles the sensitivity for detecting IGT. However, among all individuals classified as having IFG by the proposed new criteria, the proportion of individuals who also have IGT decreases to $19 \%$ compared with $28 \%$ when using the WHO criteria, thus substantially lowering the positive predictive value of IFG for predicting IGT. The proportion of individuals with IFG who have normal 2-h plasma glucose was $66 \%$ with the current WHO criteria and $78 \%$ with the proposed new ADA criteria. These findings were confirmed in the DETECT-2 data, where the proportion of individu- 
als having IGT detected by the two different diagnostic criteria for IFG increased. This increase ranged from 22 to $47 \%$ in USA and from 43 to $74 \%$ in France. However, the proportion of individuals with IFG who also had IGT fell by between $17 \%$ in China and $38 \%$ in France (Table 4). Thus, while the proposed new ADA criteria for IFG increase the sensitivity for identifying cases of IGT, it does so at the expense of a substantial increase in the proportion of individuals who would be labelled as pre-diabetic with a normal 2-h glucose value. The prognostic impact of this is currently unknown.

The sensitivity and specificity of IFG and IGT in predicting subsequent development of diabetes are important characteristics of the two glucose categories. Previous studies have shown that in a high-risk population (Pima Indians) the sensitivity of the IFG category is lower than that of the IGT category [20], while in the European, white Caucasian population the sensitivity was the same for IFG and IGT [21]. The difference in performance between IFG and IGT may reflect the fact that the WHO-defined IFG group only identifies 20 to $30 \%$ of all individuals with IGT. The 2003 ADA expert committee report [1] showed that lowering the FPG criteria for IFG to between 5.2 and $5.7 \mathrm{mmol} / \mathrm{l}$ would maximise the sensitivity and specificity in predicting the development of diabetes. In the present study we did not have follow-up data with repeated glucose tolerance tests to examine this. However, in previous analyses from the DECODE and DECODA studies we have demonstrated that the association between other established risk factors for diabetes and the presence of undiagnosed diabetes differs markedly between ethnic groups [23]. It is not unlikely that the sensitivity and specificity of fasting plasma glucose as a predictor of the development of diabetes is modified by obesity and ethnic or genetic factors related to the susceptibility for developing diabetes. This possibility should be tested in more populations before the adoption of new diagnostic criteria for IFG.

During the last 10 years at least four studies have demonstrated that the risk of developing diabetes can be reduced by 40 to $60 \%$ in individuals with IGT [7, $8,9,10]$. Over a 5 -year period of observation the incidence is delayed by 3 to 4 years. This observation, combined with the difficulties associated with identifying individuals with IGT, has raised the question of whether individuals with "prediabetes" can be identified through questionnaires, phenotypic characteristics or fasting plasma glucose. All intervention studies have been performed in individuals with IGT identified in a step-wise process from among high-risk individuals with a family history or phenotypic characteristics, and diagnosed as IGT on the basis of an OGTT. No studies have intervened strictly on the basis of fasting plasma glucose or a combination of fasting plasma glucose and phenotypic characteristics. In the recent expert committee report [1] it is stated that the majority of individuals qualifying for the intervention trials would also have IFG, and in the Diabetes Prevention Programme [7] $80 \%$ of the subjects included with IGT had FPG of 5.6 to $6.9 \mathrm{mmol} / \mathrm{l}$. This could indicate that the IFG group would be a rational target for future interventions. However, as shown by data from the Inter99 study, only $18.5 \%$ in the proposed new ADA IFG category will also have IGT, compared to $27.8 \%$ with the WHO criteria. For the three large countries together (India, China and USA) the proportion of individuals with IFG who also have IGT would decrease from $29.5 \%$ to $23.6 \%$. In other words, for every four individuals in the 40 to 64 age group recruited for intervention on the basis of IFG, one would have IGT and thus a documented 50 to $60 \%$ reduced probability of developing diabetes over the following 6 years, while the treatment effect for the remaining three individuals would be unknown and potentially non-existent or markedly lower. This seriously questions the applicability and general validity of the results from the IGT-intervention trials with regard to the revised IFG category [24]. Furthermore, the present study shows that with the proposed new criteria for IFG, the large group of individuals with isolated IFG will have markedly lower cardiovascular risk than persons identified with the WHO criteria. They will also have lower fasting and 2-hour insulin levels suggesting that they are less insulin-resistant than individuals classified as IFG on the basis of the WHO criteria. Together these observations would suggest that the proposed new isolated IFG group would be likely to have a lower incidence of diabetes and lower risk of cardiovascular disease, reducing its relevance as a target for future intervention.

In summary, the proposed new criteria for impaired fasting glycaemia suggested by the ADA expert committee will increase the prevalence of IFG two- to four-fold. IFG and IGT will continue to remain two distinctly different categories of glucose intolerance and the usefulness of IFG as a target group for evidence-based diabetes prevention strategies will become highly questionable. Based on the data from the DETECT-2 project, the likely impact on the prevalence of IFG is enormous, creating a pandemic of prediabetes, unsupported by evidence that this will positively impact on preventive actions and interventions. It is also questionable and to a large extent unknown what the prognostic impact of this labelling would be, but it cannot be ruled out that this labelling would affect the quality of life as well as finances (health insurance, life insurance etc.) of those affected.

Against this background, we would suggest that the existing criteria for IFG should remain unchanged until the impact of the proposed change on the future development of diabetes and cardiovascular disease has been assessed and there is evidence that intervening in people with IFG will actually benefit them. Until such data are available, a revision of the diagnostic criteria 
for IFG would again have to be consensus-based and not evidence-based. At a time where clinical decisions are evidence-based, this principle should also apply to public health, preventive medicine and even to the revision of diagnostic criteria. The resulting increased burden on an already overburdened health system would only be justified, if based on solid evidence.

Acknowledgements. The assistance of Dorte Vistisen, statistician at Steno Diabetes Centre, with preparation of the data set is highly appreciated. We also thank the centres which contributed data to the DETECT-2 project for their generous support in providing their original data.

\section{References}

1. The Expert Committee on the diagnosis and classification of diabetes mellitus (2003) Follow-up report on the diagnosis of diabetes mellitus. Diabetes Care 26:3160-3167

2. The Expert Committee on the diagnosis and classification of diabetes mellitus (1997) Report of the Expert Committee on the diagnosis and classification of diabetes mellitus. Diabetes Care 20:1183-1197

3. World Health Organization (1999) Definition, diagnosis and classification of diabetes mellitus and its complications. Report of a WHO consultation. Part 1: Diagnosis and classification of diabetes mellitus. World Health Organization, Geneva

4. Davidson MB, Landsman PB, Alexander CM (2003) Lowering the criterion for impaired fasting glucose will not provide clinical benefit. Diabetes Care 26:3329-3330

5. Genuth S (2003) Lowering the criterion for impaired fasting glucose is in order. Diabetes Care 26:3331-3332

6. DECODE-Study Group (1999) Is fasting glucose sufficient to define diabetes? Epidemiological data from 20 European studies. Diabetologia 42:647-654

7. Pan X, Li G, Hu Y-H et al. (1997) Effects of diet and exercise in preventing NIDDM in people with impaired glucose tolerance. The Da Qing IGT and Diabetes Study. Diabetes Care 20:537-544

8. Tuomilehto J, Lindstrom J, Eriksson JG et al. (2001) Prevention of type 2 diabetes mellitus by changes in lifestyle among subjects with impaired glucose tolerance. N Engl J Med 344:1343-1350

9. Diabetes Prevention Program Research Group (2002) Reduction in the incidence of type 2 diabetes with lifestyle intervention or metformin. N Engl J Med 346:393-403

10. Chiasson JL, Josse RG, Gomis R, Hanefeld M, Karasik A, Laakso M (2002) Acarbose for prevention of type 2 diabetes mellitus: the STOP-NIDDM randomized trial. Lancet 359:2072-2077
11. DECODE Study Group on behalf of the European Diabetes Epidemiology Study Group (1998) Will new diagnostic criteria for diabetes mellitus change the phenotype of patients with diabetes? BMJ 317:371-375

12. DECODE-Study Group (1999) Glucose tolerance and mortality. Comparison of the WHO and ADA diagnostic criteria. Lancet 354:617-621

13. Colagiuri S, Borch-Johnsen K (2003) DETECT-2: early detection of type 2 diabetes and IGT. Diabetes Voice 48:11-13

14. Glümer C, Jørgensen T, Borch-Johnsen K (2003) Prevalence of diabetes and impaired glucose regulation in a Danish population-The Inter99 study. Diabetes Care 26:2335-2340

15. Balkau B, Eschwege E, Ducimetiere P, Richard J-L, Warnet J-M (1991) The high risk of death by alcohol-related diseases in subjects diagnosed as diabetic and impaired glucose tolerance: the Paris Prospective Study after 15 years of follow-up. J Clin Epidemiol 44:465-474

16. YH Dong, WG Gao, HR Nan, for the Qingdao Epidemiology Study Group (2003) The prevalence of diabetes and the risk factor in Chinese population in Qingdao city. 18th International Diabetes Federation Congress, Paris, France, 24-29 August 2003. Diabetes Metab 29:4S21

17. Ramachandran A, Snehalatha C, Kapur A et al. (2001) High Prevalence of diabetes and impaired glucose tolerance in India. National Urban Diabetes Survey. Diabetologia 44:1094-1101

18. Harris MI, Flegal KM, Cowie CC et al. (1998) Prevalence of diabetes, impaired fasting glucose, and impaired glucose tolerance in U.S. adults. The Third National Health and Nutrition Examination Survey, 1988-1994. Diabetes Care 21:518-524

19. United Nations Population Division, Department of Economic and Social Affairs, United Nations Population Prospects. http://www.un.org/esa/population/unpop.htm, last accessed: 20/04/2004

20. Gabir MM, Hanson RL, Dabelea D et al. (2000) The 1997 American Diabetes Association and 1999 World Health Organization criteria for hyperglycaemia in the diagnosis and prediction of diabetes. Diabetes Care 23: 1108-1112

21. De Vegt F, Dekker JM, Jager A et al (2001) Relation of impaired fasting and postload glucose with incident type 2 diabetes in a Dutch population: The Hoorn Study. JAMA 285:2109-2113

22. Unwin N, Shaw J, Zimmet P, Alberti KGGM (2002) Impaired glucose tolerance and impaired fasting glycaemia: the current status on definition and intervention. Diabet Med 19:708-723

23. Nakagami T, on behalf of the DECODE-DECODA Study Group (2003) Age, body mass index and Type 2 diabetesassociations modified by ethnicity. Diabetologia 46 : 1063-1070

24. Borch-Johnsen K (2002) IGT and IFG. Time for revision? Diabet Med 19:707 